\title{
ANÁLISE DE DECISÃO MULTICRITÉRIO NA LOCALIZAÇÃO DE USINAS TERMOELÉTRICAS UTILIZANDO SIG
}

\author{
Kátia Lívia Zambon * \\ Universidade Estadual Paulista (UNESP) - Bauru - SP \\ Universidade de São Paulo (USP) - São Carlos - SP \\ katia@feb.unesp.br
}

Adriano Alber de França M. Carneiro

Antônio Nélson Rodrigues da Silva

Universidade de São Paulo (USP) - São Carlos - SP

adriano@sel.eesc.sc.usp.br; $\underline{\text { anelson@sc.usp.br }}$

\author{
Jean Cesari Negri \\ CESP - São Paulo - SP \\ jean.negri@cesp.com.br
}

* Corresponding author/autor para quem as correspondências devem ser encaminhadas

Recebido em 06/2003; aceito em 03/2005 após 2 revisões

Received June 2003; accepted March 2005 after 2 revisions

\begin{abstract}
Resumo
A integração de sistemas de informação geográfica (SIGs) e de métodos de decisão multicritério vêm proporcionando inúmeros benefícios para a resolução de problemas de planejamento e gerenciamento do mundo real. Sob esta ótica, este trabalho se propõe a avaliar as alternativas geradas em ambiente SIG para a localização de usinas termoelétricas (UTEs), aplicando técnicas de análise multicritério no processo de decisão. O modelo desenvolvido contribui para a difícil e complexa tarefa de analisar locais com potencial para a instalação de uma UTE e os diversos critérios envolvidos no problema, sejam eles de ordem econômica e/ou ambiental. A visualização dos resultados, através de mapas no SIG, mostra a aplicabilidade das técnicas de análise multicritério para o planejamento da expansão do setor elétrico. Além disso, os resultados, aplicados em um estudo de caso real, mostram cenários com situações de risco mínimo, médio (trade-off total) e máximo para uma tecnologia selecionada para a instalação da UTE.
\end{abstract}

Palavras-chave: multicritério; SIG; usinas termoelétricas.

\begin{abstract}
The combination of Geographic Information Systems (GIS) and Multicriteria Decision Analysis (MCDA) methods can bring several benefits for the solution of real-world planning and management problems. Under that view, this work aims to evaluate alternatives for the location of Thermoelectric Power Plants (TPP) produced in a GIS environment using a MCDA technique for supporting the decision-making process. The proposed model is a contribution for the TPP site selection process, which is a difficult and complex task due to the several economic and environmental criteria it involves. The visualization of the analysis outcome in GIS maps helps to demonstrate the suitability of MCDA techniques for planning the expansion of the electric supply system. In addition, the selection of a specific technology for the installation of the TPP in the real case application is used to show scenarios with different risk levels, which range from minimum to maximum risk conditions, also including the intermediate case of total trade-off.
\end{abstract}

Keywords: multicriteria; decision analysis; GIS; thermoelectric plant. 


\section{Introdução}

No setor de energia elétrica, a área que se dedica ao estudo da inserção de novos parques geradores de energia no sistema é denominada planejamento da expansão. Nesta área, as decisões de localização e instalação de novas usinas devem ser amplamente analisadas, a fim de se obter os diversos cenários proporcionados pelas alternativas geradas.

Em sistemas hidrotérmicos de potência, com predominância de geração hidráulica, como é o caso do Brasil, uma grande parte dos estudos na área de planejamento é referente à construção de usinas hidroelétricas, as quais são localizadas nos grandes rios e, em sua maioria, distantes dos centros de carga. Por este e por outros fatores, a matriz energética brasileira tende a ser gradualmente alterada pela inserção de usinas termoelétricas (UTEs).

O problema de localização de UTEs envolve um grande número de variáveis necessitando de um complexo sistema de avaliação, através do qual deve ser possível analisar a importância e contribuição de cada uma. Para a estruturação desses sistemas, os métodos de análise multicritério têm se adequado bem aos processos de tomada de decisão na área do planejamento da expansão do setor elétrico (Hobbs \& Meier, 2000; Gomes et al., 2002).

Há inúmeras variáveis espaciais envolvidas no problema de localização de UTEs, tais como: transporte de combustíveis para a operação das usinas através de ferrovias, rodovias ou gasoduto; proximidade das linhas de transmissão para o escoamento da energia elétrica gerada; e localização de áreas de preservação natural, entre outras. Todas estas compõem um rol de variáveis que necessitam de uma representação geográfica a mais próxima possível da realidade, de forma a assegurar a consistência de dados em sistemas de apoio à decisão.

Os Sistemas de Informação Geográfica (SIGs) têm sido amplamente utilizados para a estruturação e organização de variáveis espaciais na geração de alternativas para problemas dessa natureza. Seu uso intenso justifica-se pelo fato de constituir uma poderosa ferramenta que integra o conjunto de rotinas de programação desenvolvidas para representar e manipular grandes quantidades de dados armazenados em bancos de dados, os quais contêm informações representativas do mundo real, através de coordenadas geográficas, possibilitando análises espaciais (Star \& Estes, 1991).

Segundo Malczewski (1999), apesar dos SIGs e dos métodos de decisão multicritério serem duas áreas distintas de pesquisa, os problemas de planejamento e gerenciamento do mundo real podem se beneficiar da combinação de suas técnicas e procedimentos (Carver, 1991; Jankowski, 1995; Lins \& Ferreira, 1997; Gomes \& Lins, 1999). Sob esta ótica, este trabalho se propõe a avaliar as alternativas geradas em ambiente SIG, no caso o SIG-T (Sistema de Informações Geográficas para Transportes) Transcad, para a localização de UTEs aplicando técnicas de análise multicritério. O modelo desenvolvido contribui para a complexa tarefa de analisar locais com potencial para a instalação de uma UTE e os diversos critérios envolvidos no problema, sejam eles de ordem econômica ou ambiental.

Este modelo é concebido para auxiliar empreendedores que venham a ter interesse em construir uma usina, ou órgãos governamentais que possuem a função de avaliar e deferir ou não a licença de instalação e operação de usinas.

O artigo está dividido em seções, sendo que a primeira delas, após esta introdução, contém uma breve discussão acerca das variáveis abordadas no modelo desenvolvido e relacionadas ao problema de localização de UTEs (locais para a implantação da usina), além de alguns comentários acerca do uso de SIG na geração de alternativas para este tipo de problema. 
Na seção subseqüente encontra-se uma discussão de alguns métodos de análise multicritério e as premissas adotadas para as variáveis envolvidas no problema. Em seguida, é apresentada uma aplicação desses métodos em um estudo de caso real e é feita uma análise das melhores soluções obtidas através de mapas gerados no SIG. As conclusões finais baseiam-se nos métodos descritos e aplicados ao problema, bem como a constatação da viabilidade no uso de SIG e análise multicritério para a resolução de problemas de localização envolvendo variáveis espaciais.

\section{Definição de Critérios para o Problema}

Critérios são atributos que podem ser quantificados ou avaliados e que contribuem para a decisão. A busca da solução de um problema freqüentemente ocorre em ambiente onde os critérios são conflitantes, ou seja, onde o ganho de um critério poderá causar uma perda em outro. Estes critérios podem ser do tipo fator, compostos por variáveis que acentuam ou diminuem a aptidão de uma determinada alternativa para o objetivo em causa ou podem ser do tipo exclusão, variáveis que limitam as alternativas em consideração na análise, excluindo-as do conjunto solução (Mousseau, 1997).

Os modelos baseados em decisão multicritério são indicados para problemas onde existam vários critérios de avaliação. Para a localização de UTE, os critérios analisados podem ser conflitantes, já que uma usina economicamente viável não é garantia de ser ambientalmente aconselhável. Tendo isto em mente, na Figura 1 estão dispostos os critérios analisados no modelo proposto, que são discutidos com mais detalhes nos itens subseqüentes. Antes, porém, cabe aqui um comentário a respeito do SIG empregado como plataforma para as análises. O SIG-T Transcad baseia-se em uma estrutura tipo vetorial para representação de dados espaciais, através da qual as entidades são representadas por pontos, áreas ou linhas. Ao contrário de outros software que fazem uso de uma estrutura matricial (tipo raster) para este fim, a área sob análise é dividida em uma malha formada por células regulares.

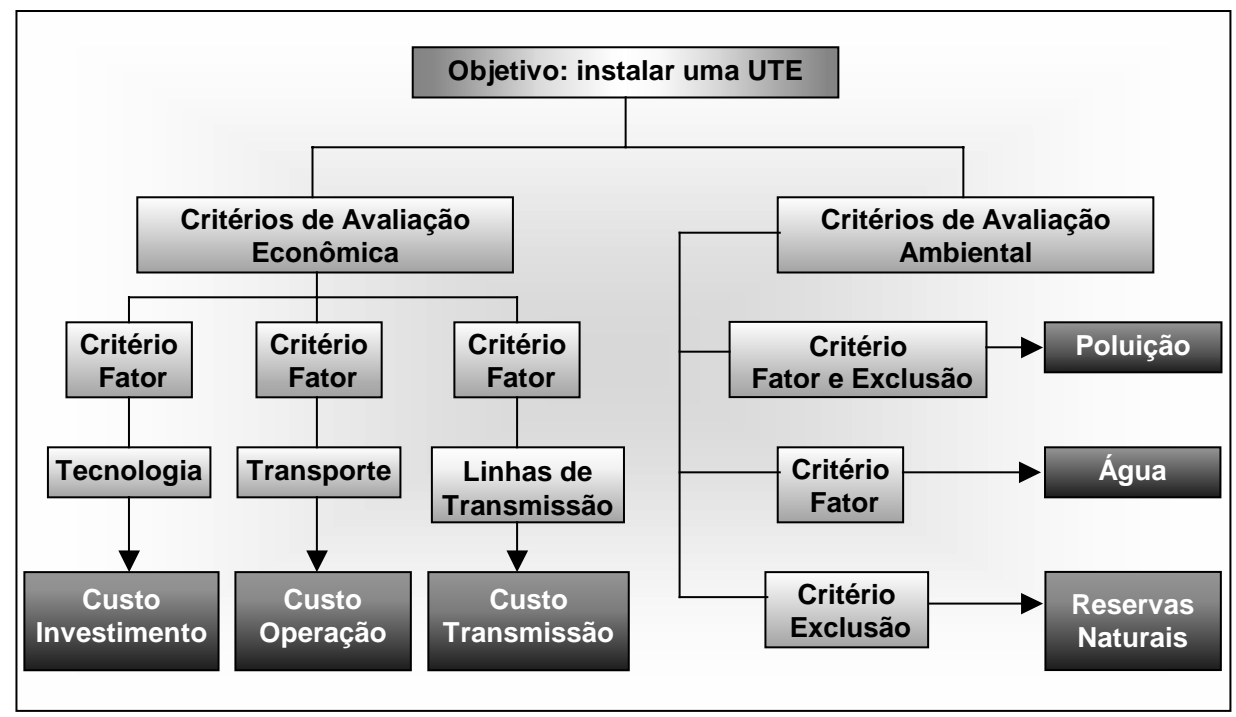

Figura 1 - Critérios adotados para a análise de localização de UTE no modelo aqui proposto. 


\subsection{Critérios econômicos}

Estes critérios contribuem para a análise econômica do problema de localização de UTE e podem ser considerados como critérios fatores, compostos por variáveis que acentuam ou diminuem a aptidão de uma determinada alternativa para o objetivo em questão. As alternativas são constituídas pelos locais com potencial para geração termoelétrica.

\subsubsection{Tecnologia}

A escolha da tecnologia a ser implantada é uma das características mais relevantes do processo de localização de UTE. Definido o tipo de tecnologia a ser utilizado, é selecionado o combustível para a geração de energia elétrica, além do tipo de transporte até o local.

Os atributos relacionados a este critério são: custo de instalação do equipamento, capacidade de produção de energia elétrica, vida útil dos equipamentos, quantidade de água necessária para resfriamento das turbinas, emissão de substâncias poluentes, etc. Alguns destes atributos serão considerados diretamente no custo do investimento do projeto e outros incidirão sobre critérios ambientais, como a poluição e disponibilidade de água do local.

O custo de investimento (CI) de uma UTE inclui os custos com equipamentos para a construção da usina $\left(\mathrm{C}_{\mathrm{inst}}\right)$, custos de importação destes equipamentos $(\mathrm{k})$, fator de recuperação de capital (FRC), capacidade de geração de energia elétrica da usina (Módulo) e potência de referência do sistema $\left(\mathrm{P}_{\text {referência }}\right)$. $\mathrm{O}$ cálculo deste custo é dado pela Equação 1 , sendo os fatores E e FRC calculados pelas Equações 2 e 3, respectivamente.

$$
\mathrm{CI}=\frac{\mathrm{C}_{\text {inst }} * \mathrm{FRC} * \mathrm{k} *\left(\frac{\text { Módulo }}{\mathrm{P}_{\text {referência }}}\right)^{0,15}}{8,76 * \frac{\mathrm{E}}{\mathrm{P}_{\text {referência }}}}
$$

Onde:

$$
\begin{aligned}
& \text { FRC }=\frac{\mathrm{i} *(1+\mathrm{i})^{\mathrm{nv}}}{\left[(1+\mathrm{i})^{\mathrm{nv}}-1\right]} \quad \begin{array}{l}
\mathrm{i}=\text { valor taxa de juros anual } \\
\mathrm{nv}=\text { vida útil do equipamento em anos }
\end{array} \\
& \mathrm{E}=\text { Fator_Capacidade_Máximo_Usina } * \mathrm{P}_{\text {referenncia }}
\end{aligned}
$$

\subsubsection{Transporte}

As ferrovias, rodovias, hidrovias e gasoduto, meios comumente utilizados para transportar os combustíveis para a operação das usinas podem ser georeferenciados no SIG utilizado como bases de dados de linhas. Como o SIG empregado é um SIG-T, inclui rotinas específicas para transporte, logo o cálculo do melhor caminho e a distância a ser percorrida entre os pontos de distribuição dos combustíveis (city-gates) até os locais (municípios) considerados na análise do problema são realizados considerando-se as condições existentes para o meio de transporte selecionado.

O custo de operação $(\mathbf{C O})$ de uma UTE é utilizado para estimar o valor a ser gasto na operação da usina, incluindo manutenção dos equipamentos, pessoal contratado para 
trabalhar em sua operação, combustíveis utilizados (custo na origem + custo de transporte), etc. Este custo é representado pela Equação 4, obtida a partir das equações 5, 6 e 7.

$$
\text { CO = Custo_básico + Custo_variável + Custo_fixo }
$$

Onde:

$$
\begin{aligned}
& \text { Custo_básico }=\frac{\text { Custo_comb }+ \text { Custo_transp }}{\text { Poder_calorífico_comb }} * \text { Fator_tecnologia } \\
& \text { Custo_variável }=\text { Valor_custo_variável_tecnologia } \\
& \text { Custo_fixo }=\frac{1000 * \text { Valor_custo_fixo_tecnologia }}{8760 * \text { Fator_capacidade_médio }}
\end{aligned}
$$

\subsubsection{Linhas de transmissão (LTs)}

Os diferentes tipos de tensão das linhas de transmissão podem ser incorporados às bases de dados de linhas e georeferenciadas no SIG. Neste caso faz-se uso das linhas de tensão de $138 \mathrm{kV}$ e $440 \mathrm{kV}$. O uso de SIG neste caso permite que seja identificada qual a tensão da linha mais próxima que abastece o município e a distância para cálculo do custo de conexão à linha que transportará a energia elétrica gerada pela UTE.

O custo unitário do benefício da transmissão (CT) é dado pelo custo de conexão às LTs (Custo_conexão) mais um crédito pela redução de perdas devido à geração local (Delta_perda), ou seja, da usina termoelétrica estar gerando energia próxima ao centro de carga. $\mathrm{O}$ custo é apresentado na Equação 8.

$$
\mathrm{CT}=\frac{\text { (Delta_perda) }-(\text { Valor_marginal })}{\mathrm{P}_{\text {referência }}}+\text { Custo_conexão }
$$

Onde:

$$
\text { Custo_conexão }=\frac{\text { Custo_138kV_ou_440kV } * 10^{6} * \text { FRC }}{8760 * \text { Fator_capacidade_médio } * \mathrm{P}_{\text {referência }}}
$$

\subsection{Critérios ambientais}

Neste trabalho são apresentados três critérios de avaliação ambiental: o nível de poluição do ar no local versus o nível de poluição emitido pela tecnologia (critério fator e de exclusão, onde alternativas de níveis de poluição inaceitáveis são excluídas do conjunto solução); a disponibilidade local de água versus a quantidade de água requerida (critério fator) e as reservas naturais (critério de exclusão), consideradas áreas de preservação ambiental (APA) e que não podem ser modificadas por ações do homem.

\subsubsection{Poluição do ar}

O estudo de caso ao qual foi aplicado o modelo aqui proposto é referente aos 645 municípios do estado de São Paulo. Considerando a inexistência de um sistema de medição para a estimativa da poluição em todos os municípios, a abordagem descrita a seguir foi utilizada. 
- Dos Municípios: Através de dados de combustíveis automotivos e industriais (gasolina, diesel, óleo combustível, querosene, GLP e outros) cedidos pela ANP (Agência Nacional de Petróleo) foi possível classificar os municípios de acordo com seu nível de poluição, utilizando para isto uma Rede Neural Artificial (RNA) com a técnica Self Organizing Map (SOM). Os 645 municípios foram classificados em 5 níveis de poluição e um mapa relacionando municípios com características semelhantes quanto à emissão de dióxido de enxofre foi gerado. Maiores detalhes encontram-se disponíveis em Zambon et al. (2003). Outro exemplo de adequação da técnica RNA-SOM para efeito de classificação pode ser visto em Mussone \& Sala (2001).

- Da Tecnologia: são informações técnicas contidas no banco de tecnologias de PROMON (1996) com índice de poluição previamente atribuído quanto aos poluentes emitidos.

\subsubsection{Disponibilidade de água}

Este critério refere-se à disponibilidade de água existente nos rios dos municípios em relação ao requisito de água exigido pela tecnologia selecionada. O processo de verificação para análise da seleção de alternativas foi realizado da seguinte forma:

- Dos Municípios: Adotou-se a vazão das 22 Unidades de Gerenciamento de Recursos Hídricos (UGRHI), que mapeiam todo o Estado, extrapolando-se os valores para os municípios pertencentes a cada Unidade. Observa-se que a utilização de uma base de dados georeferenciada dos rios do Estado seria facilmente aplicada ao procedimento, desde que houvesse disponibilidade de dados sobre vazões dos rios em cada município.

- Da Tecnologia: o requisito de água é um dado constante do banco de dados de tecnologias de PROMON (1996).

\subsubsection{Reservas naturais}

Para este critério de exclusão foram identificadas e georeferenciadas as reservas naturais que compõem o conjunto de áreas de preservação ambiental, onde não podem ser construídas as UTEs. Os valores de referência para este critério serão zero (área de preservação) ou um (não é área de preservação).

\section{Análise Multicritério}

A partir da estruturação do modelo, considerando o objetivo a ser alcançado e definidos os critérios necessários para a solução do problema, é possível utilizar as técnicas de decisão multicritério para sua resolução. Os passos podem ser assim resumidos: definição de pesos para os critérios, normalização e combinação dos critérios (Ramos, 2000).

\subsection{Definição de pesos para os critérios}

Para Ramos (2000) não há um método consensual para a definição de pesos, mas várias propostas de procedimentos para este efeito podem ser encontradas na literatura (von Winterfeltdt \& Edwards, 1986; Malczewski, 1999). 
É possível agrupar os métodos de definição de pesos em quatro categorias assim declaradas: métodos baseados em ordenação de critérios (Stillwell et al., 1981), em escalas de pontos (Osgood et al., 1957), em distribuição de pontos (Easton, 1973) e comparação de critérios par a par (Saaty, 1977).

O método aplicado neste trabalho é o de comparação par a par no contexto do processo de tomada de decisão denominado AHP (Analytic Hierarchy Process). Esta técnica baseia-se numa matriz quadrada $\mathrm{n} \times \mathrm{n}$, onde as linhas e colunas correspondem aos $\mathrm{n}$ critérios analisados para o problema em questão. Assim, o valor $\mathrm{a}_{\mathrm{ij}}$ representa a importância relativa do critério da linha i face ao critério da coluna j. Como esta matriz é recíproca, apenas a metade triangular inferior necessita ser avaliada, já que a outra metade deriva desta e a diagonal principal assume valores iguais a 1 .

O estabelecimento de comparações par a par para todos os critérios necessita da definição de uma escala, destinada à normalização. Na Tabela 1 e na Figura 2 é apresentada a escala adaptada de Saaty (1980).

Tabela 1 - Escala de comparação de critérios.

\begin{tabular}{|c|c|c|}
\hline Valor & Definição & Explicação \\
\hline 1 & Igual importância & $\begin{array}{l}\text { os dois critérios contribuem de forma idêntica } \\
\text { para o objetivo }\end{array}$ \\
\hline 3 & Pouco mais importante & $\begin{array}{l}\text { a análise e a experiência mostram que um critério } \\
\text { é um pouco mais importante que o outro }\end{array}$ \\
\hline 5 & Muito mais importante & $\begin{array}{l}\text { a análise e a experiência mostram que um critério } \\
\text { é claramente mais importante que o outro }\end{array}$ \\
\hline 7 & Bastante mais importante & $\begin{array}{l}\text { a análise e a experiência mostram que um dos } \\
\text { critérios é predominante para o objetivo }\end{array}$ \\
\hline 9 & Extremamente mais importante & $\begin{array}{l}\text { sem qualquer dúvida um dos critérios é } \\
\text { absolutamente predominante para o objetivo }\end{array}$ \\
\hline 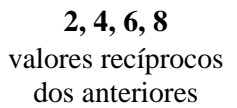 & Valores intermediários & também podem ser utilizados \\
\hline
\end{tabular}

Fonte: adaptado de Saaty (1980).

\begin{tabular}{|c|c|c|c|c|c|c|c|c|}
\hline $1 / 9$ & $1 / 7$ & $1 / 5$ & $1 / 3$ & 1 & 3 & 5 & 7 & 9 \\
\hline $\begin{array}{c}\text { extrema- } \\
\text { mente }\end{array}$ & bastante & muito & pouco & igual & pouco & muito & bastante & $\begin{array}{c}\text { extrema- } \\
\text { mente }\end{array}$ \\
\hline \multicolumn{5}{|c|}{ MENOS IMPORTANTE } & \multicolumn{4}{|c|}{ MAIS IMPORTANTE } \\
\hline
\end{tabular}

Figura 2 - Escala de comparações de critérios.

Os pesos obtidos para os testes propostos neste trabalho foram definidos pela técnica AHP analisando-se o grau de consistência (CR - Consistency Ratio) resultante do processo. Segundo Saaty \& Vargas (1991) quando o CR é superior a 0,1 é necessário reavaliar o processo. Esta afirmação foi feita baseando-se em vários contextos em que se utilizou o 
método AHP para a resolução de diferentes tipos de problemas complexos. Para o problema da localização de UTEs, após uma extensiva análise, a matriz de comparação par a par obtida para os critérios do tipo fator selecionados (Figura 1) é apresentada na Tabela 2.

Tabela 2 - Matriz de comparação par a par.

\begin{tabular}{|l|c|c|c|}
\cline { 2 - 4 } \multicolumn{1}{c|}{} & Custos & Nível de Poluição & Água \\
\hline Custos & $\mathbf{1}$ & $\mathbf{1 / 8}$ & $\mathbf{2}$ \\
\hline Nível de Poluição & $\mathbf{8}$ & $\mathbf{1}$ & $\mathbf{9}$ \\
\hline Agua & $\mathbf{1 / 2}$ & $\mathbf{1 / 9}$ & $\mathbf{1}$ \\
\hline
\end{tabular}

Após todos os cálculos necessários para a obtenção do grau de consistência (CR) e as reavaliações das opções adotadas, o valor definido para CR foi de 0,063867 . Os pesos obtidos através deste processo estão na Tabela 3. Os custos de investimento, operação e transmissão foram agrupados para este estudo, sendo valores fornecidos em US $\$ / \mathrm{MWh}$. A ordem de prioridade identificada pelo valor dos pesos relata a realidade do problema em relação a este tipo de localização.

Tabela 3 - Pesos definidos para os critérios fatores do problema.

\begin{tabular}{|l|c|}
\hline \multicolumn{1}{|c|}{ Critério Fator Analisado } & Peso Obtido \\
\hline Nível de Poluição & $\mathbf{0 , 8 0 4 4}$ \\
\hline Custos (CI + CO + CT) & $\mathbf{0 , 1 2 2 0}$ \\
\hline Disponibilidade de Água & $\mathbf{0 , 0 7 3 6}$ \\
\hline
\end{tabular}

\subsection{Normalização de critérios}

Este processo permite que valores de critérios não comparáveis entre si sejam normalizados para uma mesma escala, viabilizando a agregação entre eles. A maior parte dos processos de normalização utiliza o valor máximo e mínimo para a definição de uma escala. A forma mais simples é uma variação linear definida pela Equação 10 (Eastman, 1997).

$$
\mathrm{x}_{\mathrm{i}}=\frac{\mathrm{R}_{\mathrm{i}}-\mathrm{R}_{\min }}{\mathrm{R}_{\max }-\mathrm{R}_{\min }} * \text { IntervaloNormalizado }
$$

Onde:

$$
\begin{aligned}
& \mathrm{R}_{\mathrm{i}} \text { : é o valor a ser normalizado } \\
& \mathrm{R}_{\min } \text { : valor mínimo para o critério } \\
& \mathrm{R}_{\max } \text { : valor máximo para o critério }
\end{aligned}
$$

O processo de normalização é na sua essência idêntico ao processo de fuzzification introduzido pela lógica fuzzy, segundo o qual um conjunto de valores pode ser expresso (convertido) numa escala normalizada (por exemplo, entre zero e um), tornando-os comparáveis. Para este processo existem várias funções fuzzy que podem ser utilizadas, sendo as mais conhecidas: sigmoidal, j-shaped, linear e complexa (Zadeh, 1965). 
A função linear utilizada para a normalização do critério referente aos custos e disponibilidade de água é apresentada na Figura 3.

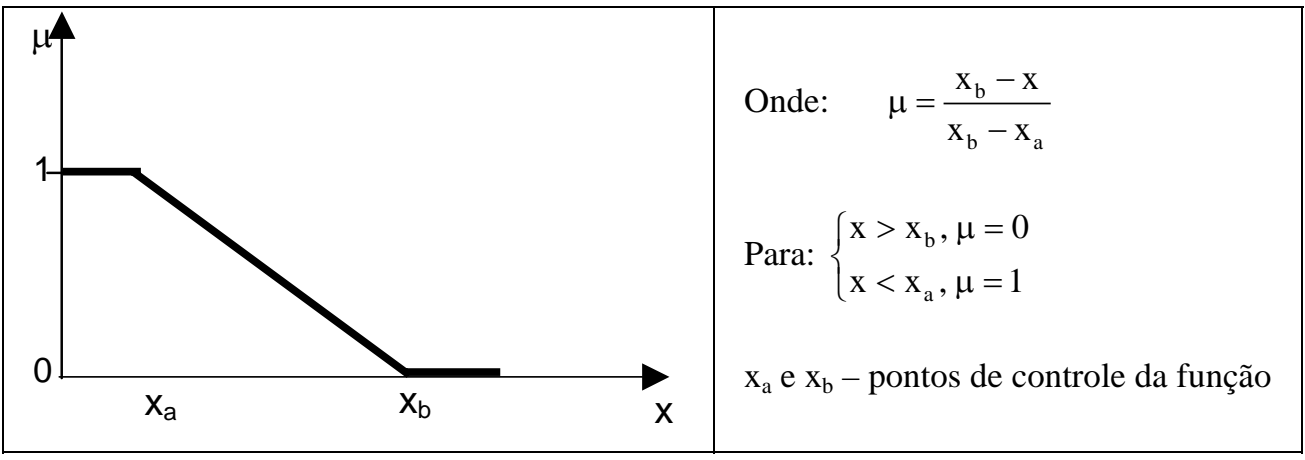

Figura 3 - Função fuzzy.

Para a normalização do critério nível de poluição, a Tabela 4 é utilizada para expressar os valores dos níveis de poluição dos municípios e da tecnologia a ser selecionada. Por exemplo, para uma tecnologia que possui nível de poluição igual a 2 e um município com nível de poluição I, o valor normalizado é dado por 0,90.

Tabela 4 - Normalização para o critério nível de poluição.

\begin{tabular}{|c|c|c|c|c|c|}
\hline $\begin{array}{c}\text { Tecnologia } \rightarrow \\
\text { Município } \downarrow\end{array}$ & $\mathbf{1}$ & $\mathbf{2}$ & $\mathbf{3}$ & $\mathbf{4}$ & $\mathbf{5}$ \\
\hline I & 1,00 & 0,90 & 0,80 & 0,60 & 0,40 \\
\hline II & 0,90 & 0,75 & 0,60 & 0,40 & 0,30 \\
\hline III & 0,80 & 0,60 & 0,50 & 0,20 & 0,15 \\
\hline IV & 0,60 & 0,40 & 0,20 & 0,25 & 0,00 \\
\hline V & 0,40 & 0,30 & 0,15 & 0,00 & 0,00 \\
\hline
\end{tabular}

\subsection{Combinação de critérios}

Depois que os scores dos critérios forem normalizados para uma escala de zero a um (ou outra qualquer), é possível agregá-los de acordo com a regra de decisão. Em Malczewski (1999) há uma descrição extensiva de classes de operadores para este fim. Dois métodos são utilizados neste trabalho, o da Combinação Linear Ponderada (WLC, derivado do inglês Weighted Linear Combination) e o da Média Ponderada Ordenada (OWA, do inglês Ordered Weighted Average).

Quando há critérios do tipo fator e exclusão, caso em que se insere o problema da localização de UTEs, o procedimento WLC é dado pela Equação 11, onde o score de cada alternativa é calculado com base nos critérios normalizados dos fatores e multiplicado pelo produtório dos critérios exclusão, representados em escala binária, 0 ou 1. 


$$
\mathrm{S}=\sum_{\mathrm{i}=1}^{\mathrm{n}} \mathrm{w}_{\mathrm{i}} \mathrm{x}_{\mathrm{i}} * \prod_{\mathrm{j}=1}^{\mathrm{k}} \mathrm{c}_{\mathrm{j}}
$$

Onde:

S: valor final do score

$\mathrm{w}_{\mathrm{i}}$ : peso do critério fator $\mathrm{i}(\operatorname{com} \mathrm{i}=1, \ldots, \mathrm{n})$

$\mathrm{n}$ : número de critérios fator

$\mathrm{x}_{\mathrm{i}}$ : valor normalizado para o mesmo fator

$\mathrm{c}_{\mathrm{j}}$ : score (0 ou 1$)$ do critério exclusão $(\operatorname{com} \mathrm{j}=1, \ldots, \mathrm{k})$

k: número de critérios exclusão

Os dois critérios de exclusão neste modelo são referentes às áreas de preservação ambiental e nível de poluição (critério fator e também exclusão). Este último possui uma característica muito particular, sendo um critério fator e também incluído no produtório da Equação 11 como um critério exclusão. Esta adaptação é para os casos em que se inserem municípios com elevados níveis de poluição, aqueles que, quando normalizados, são iguais a zero.

\section{Alternativas para a Localização - Estudo de Caso Real}

A tecnologia selecionada para a aplicação do modelo desenvolvido é uma turbina a gás, que opera em ciclo combinado, referida por TG-CC. Nela, o combustível é queimado em uma ou mais turbinas a gás, gerando energia elétrica. Os gases queimados que deixam a turbina a gás são aproveitados em uma caldeira de recuperação para gerar vapor e acionam uma turbina a vapor. Como o vapor é obtido sem a queima de combustível, a potência acrescentada pela turbina a vapor não tem um correspondente consumo de combustível, o que faz do ciclo combinado o meio mais eficiente para se gerar energia elétrica termicamente (PROMON, 1996).

Esta tecnologia é operada com o combustível gás natural da Bolívia, sendo seu transporte realizado por gasoduto.

\subsection{Aplicação da técnica WLC}

Os valores associados para os 645 municípios do estado de São Paulo foram inseridos no banco de dados da base de pontos que representa os centros geográficos dos municípios. Na Tabela 5 são apresentados alguns valores (scores) obtidos para a tecnologia TG-CC com a aplicação da técnica WLC (Equação 11). Os scores estão dispostos em ordem decrescente, iniciando com as melhores opções para instalar a UTE e finalizando com os municípios excluídos da solução, que apresentam valores iguais a zero. Os atributos normalizados na referida tabela são:

Custos: os valores econômicos referentes ao custo de investimento do projeto (CI), custo de operação da tecnologia em cada município $(\mathrm{CO})$ e o custo de transmissão de energia em cada município (CT), formaram um valor representativo de todo o custo e foram normalizados, onde o valor 1 da escala corresponde a um projeto de baixo custo e o valor 0 a um de alto custo. 
Poluição: a este critério foram atribuídos valores normalizados da Tabela 4, sendo a tecnologia TG-CC de nível 2.

Água: o requisito de água para TG-CC é de $7,5 \mathrm{~m}^{3} / \mathrm{s}$, sendo as disponibilidades de água dos municípios normalizadas através da função dada pela Equação 12. Nesta equação, os pontos de controle são de valor mínimo $\left(7,5 \mathrm{~m}^{3} / \mathrm{s}\right)$, correspondente ao requisito de água da tecnologia selecionada, e de valor máximo $\left(157 \mathrm{~m}^{3} / \mathrm{s}\right)$, vazão máxima encontrada nas 22 UGRHI.

$$
\mu=\frac{\text { (Dispon_agua) }-7,5}{157-7,5} \quad \text { para: }\left\{\begin{array}{l}
\mathrm{x}>157, \mu=1 \\
\mathrm{x}<7,5, \mu=0
\end{array}\right.
$$

Reservas Naturais (APAs): são locais que receberam valor igual a zero da escala binária e que não podem receber nenhum tipo de UTE. Para locais que não se incluem nesta categoria, o valor associado é igual a 1.

Score Final: este valor identificará, numa escala decrescente, o melhor ou melhores locais (valores próximos a 1) para a instalação da tecnologia TG-CC, segundo a definição de pesos apresentada na seção 3.1.1. A Equação 11 foi aplicada e é apresentada com os critérios e pesos associados.

$$
\text { Score_Final }=(\text { Custos*0,1220 + Poluição*0,8044 + Água*0,0736 }) * \text { APA*Poluição }
$$

\begin{tabular}{|c|c|c|c|c|c|c|c|c|c|c|}
\hline \multirow[b]{2}{*}{ ID } & \multirow[b]{2}{*}{ Município } & \multirow[b]{2}{*}{$\begin{array}{l}\text { Lati- } \\
\text { tude }\end{array}$} & \multirow[b]{2}{*}{$\begin{array}{l}\text { Longi- } \\
\text { tude }\end{array}$} & \multirow[b]{2}{*}{$\begin{array}{l}\text { Altitu- } \\
\text { de }(\mathbf{m})\end{array}$} & \multirow[b]{2}{*}{$\begin{array}{l}\text { Dist. (km) } \\
\text { Gasoduto }\end{array}$} & \multicolumn{4}{|c|}{$\begin{array}{l}\text { Valores Normalizados } \begin{array}{r}\text { Escala } 1 \rightarrow 0 \\
(\text { Alternativa mais apta } \rightarrow\end{array} \text { menos apta) }\end{array}$} & \multirow[b]{2}{*}{$\begin{array}{l}\text { Score } \\
\text { Final }\end{array}$} \\
\hline & & & & & & Custos & $\begin{array}{l}\text { Polui- } \\
\text { ção }\end{array}$ & Água & APA & \\
\hline 1 & Mongaguá & $-24,08$ & $-46,62$ & 10 & 11,20 & 0,9708 & 0,90 & 1,00 & 1,00 & 0,8244 \\
\hline 2 & Ribeira & $-24,65$ & $-49,00$ & 160 & 16,78 & 0,9029 & 0,90 & 1,00 & 1,00 & 0,8169 \\
\hline 3 & Taquarivaí & $-23,92$ & $-48,69$ & 640 & 0,99 & 0,9004 & 0,90 & 1,00 & 1,00 & 0,8167 \\
\hline 4 & Rafard & $-23,01$ & $-47,52$ & 520 & 3,36 & 0,8897 & 0,90 & 1,00 & 1,00 & 0,8155 \\
\hline 5 & Mombuca & $-22,92$ & $-47,56$ & 540 & 2,84 & 0,8881 & 0,90 & 1,00 & 1,00 & 0,8153 \\
\hline 6 & Itaoca & $-24,64$ & $-48,84$ & 150 & 24,04 & 0,8842 & 0,90 & 1,00 & 1,00 & 0,8149 \\
\hline . & . & $\cdot$ & $\cdot$ & $\cdot$ & $\cdot$ & $\cdot$ & . & • & $\cdot$ & $\cdot$ \\
\hline . & $\cdot$ & $\cdot$ & $\cdot$ & $\cdot$ & $\cdot$ & $\cdot$ & $\cdot$ & • & - & $\cdot$ \\
\hline 645 & Tupi Paulista & $-21,38$ & $-51,57$ & 400 & 20,92 & 0,7507 & 0,90 & 1,00 & 0,00 & 0,0000 \\
\hline
\end{tabular}

Tabela 5 - Dados representativos para a localização da tecnologia TG-CC - técnica WLC.

Os valores de scores mais altos (próximos de 1) selecionaram os locais mais aptos a receber a tecnologia proposta. A influência maior está relacionada aos custos da usina (investimento, operação e transmissão), já que as 6 primeiras alternativas possuem o mesmo valor normalizado para os outros critérios em questão (poluição, água e APAS). Também se pode observar no mapa da Figura 4 que as melhores alternativas estão próximas ao gasoduto, aumentando a aptidão para o custo de operação da usina, neste caso, dependente do gasoduto. 


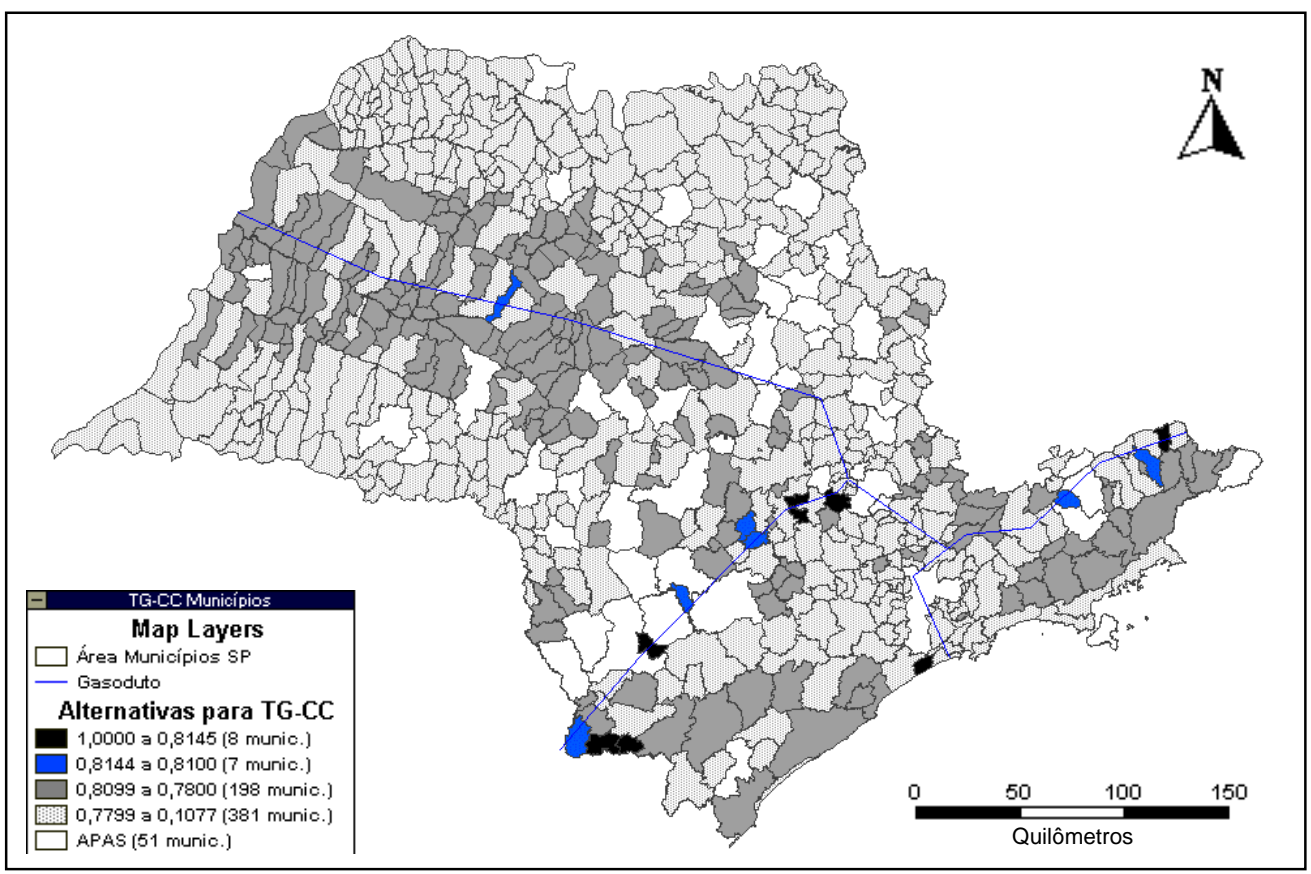

Figura 4 - Mapa das alternativas para a tecnologia TG-CC - Decisão Espacial Multicritério.

Outros cenários poderão ser analisados com a diferenciação dos pesos entre os custos, com o objetivo de avaliar, por exemplo, a contribuição do benefício da transmissão em localizar a UTE próxima a um centro de carga.

\subsection{Combinação de critérios - técnica OWA}

Esta técnica, além de utilizar os pesos de critérios utilizados no procedimento WLC, considera a aplicação de um novo conjunto de pesos denominados order weights. Estes pesos não estão associados aos critérios, mas sim à ordem que lhes é atribuída depois da aplicação da técnica WLC. Através desta agregação de critérios é possível analisar as diferentes situações de risco que podem envolver um empreendimento (Yager, 1988).

Com a variação dos conjuntos de pesos, num processo de decisão que envolve 3 critérios fatores, neste trabalho caracterizado por custos, poluição e disponibilidade de água, com valores normalizados, as seguintes situações são analisadas:

- análise de risco máximo: aplicando um conjunto de pesos $\left[\begin{array}{lll}0 & 0 & 1\end{array}\right]$ ao critério com o maior score, produzindo assim uma solução dita otimista. Este processo foi realizado para os 645 municípios sob investigação e exemplificado para o município de Adamantina na Tabela 6 ( $1^{\mathrm{a}}$ fase) e na Tabela 7 ( $2^{\mathrm{a}}$ fase). O resultado para todos os municípios pode ser visualizado no mapa (a) da Figura 5.

- análise de risco mínimo: aplicando um conjunto de pesos [1 0 l 0 0] ao critério com o menor score, produzindo assim uma solução dita pessimista ou conservadora. Para todos os municípios, o resultado pode ser visualizado no mapa (b) da Figura 5. 
- análise de risco médio: aplicando um conjunto de pesos [0,333 $0,333 \quad 0,333]$ perfeitamente equilibrado a todos os critérios, permitindo a compensação entre eles (trade-off total). A verificação pode ser visualizada no mapa (c) da Figura 5.

Tabela 6 - Dados para aplicação da técnica OWA ( $1^{a}$ fase $)$ - Município Adamantina.

\begin{tabular}{|c|c|c|c|c|}
\cline { 2 - 5 } \multicolumn{1}{c|}{} & Critério & $\begin{array}{c}\text { Valores } \\
\text { Normalizados }\end{array}$ & Pesos & $\begin{array}{c}\text { Avaliação } \\
(\mathbf{1} \text { a . fase) }\end{array}$ \\
\hline A & Custo & 0,7398 & 0,1220 & 0,0902 \\
\hline B & Poluição & 0,7500 & 0,8044 & 0,6033 \\
\hline C & Água & 1,0000 & 0,0736 & 0,0736 \\
\hline
\end{tabular}

Fazendo variar os order weights, este procedimento permite implementar uma gama vastíssima de operadores de agregação.

Tabela 7 - Dados para aplicação da técnica OWA ( $2^{\text {a }}$ fase $)$ - Município Adamantina.

\begin{tabular}{|c|c|c|c|c|}
\hline Critério & $\begin{array}{c}\text { Avaliação } \\
\left(1^{\mathrm{a}} \text { fase }\right)\end{array}$ & Order Weight & $\begin{array}{l}\text { Avaliação } \\
\left(2^{\mathrm{a}} \text { fase }\right)\end{array}$ & $\begin{array}{c}\text { Score final } \\
\text { (soma ponderada) }\end{array}$ \\
\hline B & 0,6033 & 1 & 0,6033 & \multirow{3}{*}{$\begin{array}{c}\mathbf{0 , 6 0 3 3} \\
\text { (Risco Máximo) }\end{array}$} \\
\hline $\mathbf{A}$ & 0,0902 & 0 & 0 & \\
\hline C & 0,0736 & 0 & 0 & \\
\hline B & 0,6033 & 0 & 0 & \multirow{3}{*}{$\begin{array}{c}\mathbf{0 , 0 7 3 6} \\
\text { (Risco Mínimo) }\end{array}$} \\
\hline $\mathbf{A}$ & 0,0902 & 0 & 0 & \\
\hline C & 0,0736 & 1 & 0,0736 & \\
\hline B & 0,6033 & 0,333 & 0,2009 & \multirow{3}{*}{$\begin{array}{c}\mathbf{0 , 2 5 5 4} \\
\text { (Risco Médio- } \\
\text { Trade-off Total) }\end{array}$} \\
\hline $\mathbf{A}$ & 0,0902 & 0,333 & 0,0300 & \\
\hline C & 0,0736 & 0,333 & 0,0245 & \\
\hline
\end{tabular}

A partir dos três mapas da Figura 5 é possível verificar no mapa (c) que as melhores alternativas para a implantação da tecnologia TG-CC coincidiram com a técnica WLC, apresentada no mapa da Figura 4. Isto porque o procedimento WLC é um caso particular do procedimento mais geral OWA, onde há compensação entre os critérios. No mapa (a) as alternativas ordenadas como melhores soluções consideram o critério com maior valor para a atribuição do maior peso, o qual depois de normalizado ( $1^{\mathrm{a}}$ fase, de acordo com a ordem no ranking) coincidiram com a poluição. No mapa (b) as alternativas com maior score são provenientes do critério com valor mais baixo, atribuindo a este o maior peso, coincidindo na maior parte dos casos, com a disponibilidade de água. 


\section{Conclusões}

O problema da localização de UTEs é caracterizado por um grande número de variáveis que compõem os critérios relevantes ao processo de decisão dos locais com potencial para a instalação da usina. Estes critérios são, em sua maioria, considerados conflitantes, como os de ordem econômica e os de ordem ambiental. Além disso, são fornecidos por diferentes setores e em geral, não são padronizados para efeito de comparação entre si.

Para a resolução deste problema, o modelo aqui proposto integra as componentes espaciais do problema (municípios, ferrovias, rodovias, gasoduto, linhas de transmissão) através de um SIG-T, na fase de geração de alternativas, e incorpora as técnicas de análise multicritério na fase de ordenação das alternativas válidas para a instalação da UTE com a tecnologia selecionada.

No planejamento da expansão do setor elétrico é especialmente importante o uso de ferramentas de apoio à decisão e, por este motivo, o modelo proposto pode ser utilizado por empresas do setor energético ou órgãos responsáveis pelo licenciamento de empreendimentos deste tipo. Neste sentido, foi objetivo deste trabalho a introdução de dados reais nos testes propostos para efeitos de comparação e análise para diferentes tecnologias capazes de gerar energia elétrica através das UTEs.

A análise multicritério, aplicada às diferentes técnicas de avaliação, proporcionou ao modelo consistência nos processos de ordenação das alternativas. A técnica WLC tem a importante característica de permitir a compensação entre os critérios (trade-off), o que significa que um critério com valor baixo pode ser compensado por outros com valores mais altos no processo de agregação. Pela técnica OWA é possível avaliar diversos cenários em que situações como a de risco mínimo, médio e máximo possam ocorrer, sendo os scores extremos considerados nas análises de risco máximo e mínimo, o que significa que os critérios não podem ser compensados uns pelos outros (ausência de trade-off).

Na aplicação destas técnicas podem ser observados os resultados obtidos e apresentados nas Figuras 4 e 5, aplicados para o estado de São Paulo. Na Figura 4 os oito primeiros municípios selecionados apresentaram características de baixo custo (influenciados pelo custo de operação por serem municípios localizados próximos ao meio de transporte: gasoduto), baixa poluição e com disponibilidade de água suficiente para a instalação da UTE sob análise. Estes mesmos municípios estão selecionados no mapa (c) da Figura 5, onde há uma compensação dos pesos para os critérios analisados.

A utilização da ferramenta SIG nos processos de localização em geral tem se mostrado eficiente, contribuindo para o desenvolvimento de modelos como o proposto neste trabalho. A incorporação de técnicas de decisão multicritério para o aprimoramento dos resultados obtidos no SIG tem trazido inúmeros benefícios para as avaliações do planejamento da expansão no setor elétrico.

O modelo proposto é aplicável em qualquer sistema ou região com a substituição dos dados correspondentes à área desejada, alimentando os bancos de dados, o que para o setor de energia é fundamental. Além disso, novos critérios, pesos e parâmetros podem ser incorporados ao trabalho sem prejuízo da modelagem aqui proposta. 

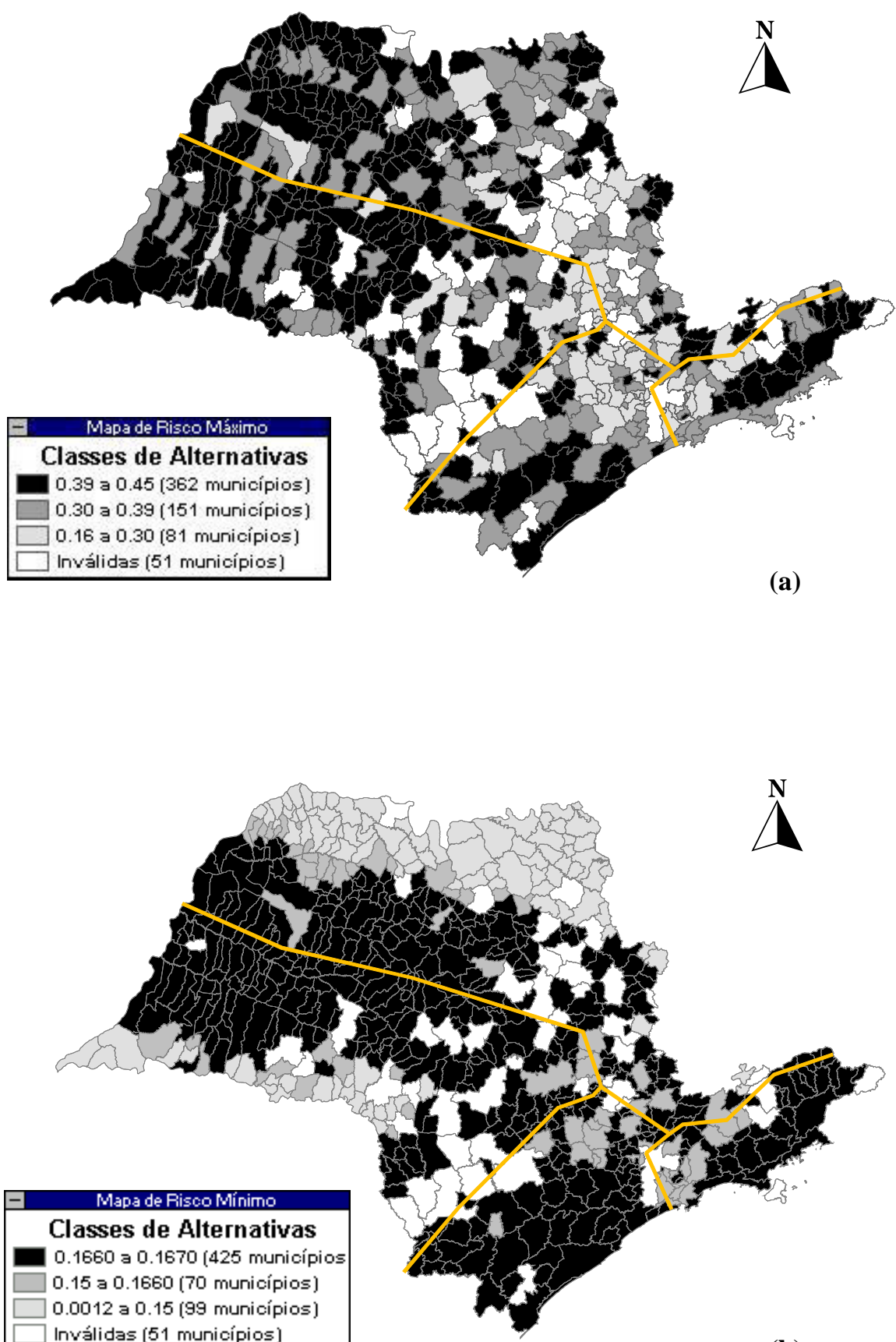

(b) 


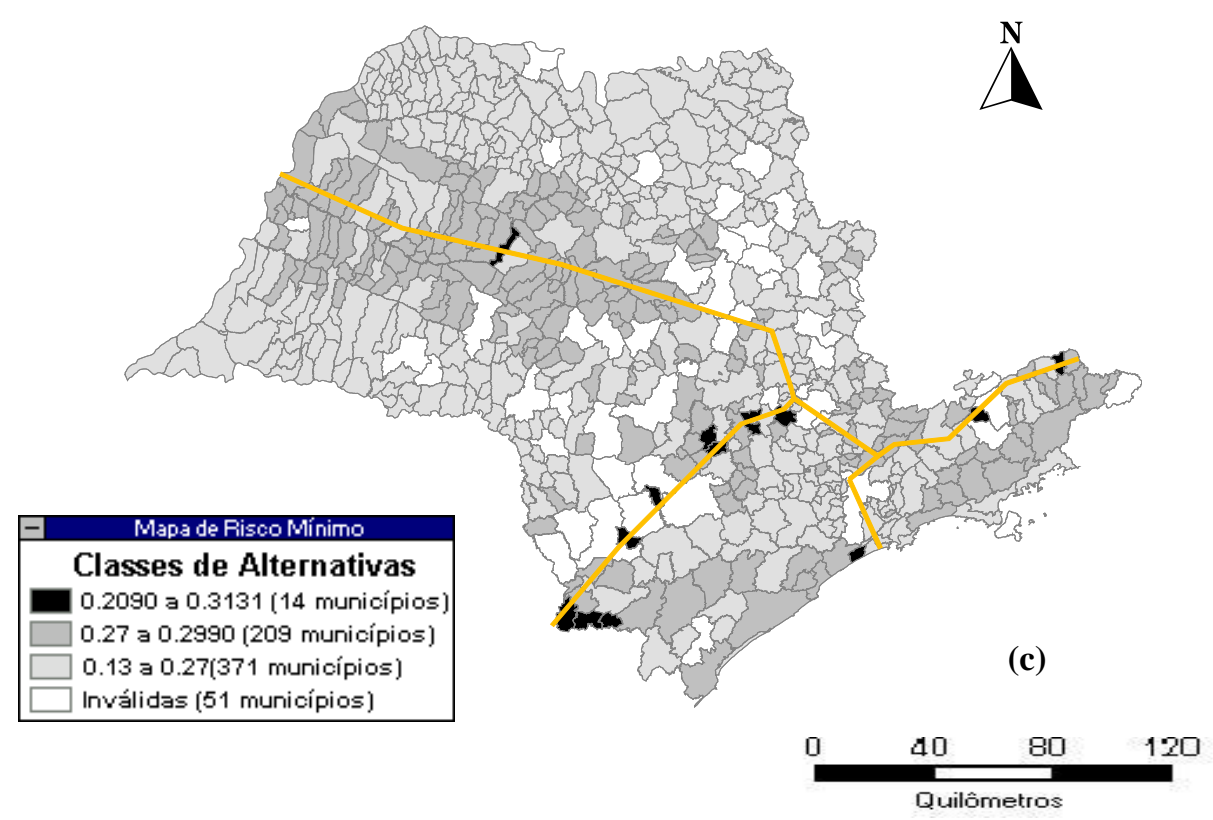

Figura 5 - Mapas de análise de risco para os critérios abordados - técnica OWA.

\section{Agradecimentos}

Os autores agradecem as empresas colaboradoras: CESP, CTEEP, AES-Tietê, Ferroban, FerroNorte, Novoeste, Cetesb, Seade, ANP. Pelo apoio financeiro, ao CNPq, Fundunesp e FAPESP.

\section{Referências Bibliográficas}

(1) Carver, S.J. (1991). Integrating multi-criteria evaluation with geographical information systems. International Journal of Geographical Information System, 5(3), 321-339.

(2) Eastman, J.R. (1997). IDRISI for Windows: User's Guide. Version 2.0. Clark University - Graduate School of Geography, Worcester, MA, USA.

(3) Easton, A. (1973). Complex Managerial Decision Involving Multiple Objectives. John Wiley \& Sons, New York, NY, USA.

(4) Gomes, E.G. \& Lins, M.P.E. (1999). Integração entre Sistema de Informação Geográfica e Métodos de Análise Multicritério no Apoio à Decisão Espacial. Anais do XXXI SBPO - Simpósio Brasileiro de Pesquisa Operacional, Juiz de Fora - MG, outubro de 1999.

(5) Gomes, L.F.A.M.; Almeida, A.T. \& Gomes, C.F.S. (2002). Tomada de Decisão Gerencial - Enfoque Multicritério. Editora Atlas, São Paulo.

(6) Hobbs, B.F. \& Meier, P. (2000). Energy Decisions and the Environment - a Guide to the Use of Multicriteria Decisions. Kluwer Academic Publishers, Boston. 
(7) Jankowski, P. (1995). Integrating geographical information systems and multiple criteria decision-making methods. International Journal of Geographical Information System, 9, 251-273.

(8) Lins, M.P.E. \& Ferreira Filho, V.J.M. (1997). Sistemas de Informação Geográfica e Modelagem em PO. Revista Pesquisa Operacional, 17(2), 178-187.

(9) Malczewski, J. (1999). GIS and Multicriteria Decision Analysis. John Wiley \& Sons, New York.

(10) Mousseau, V. (1997). Compensatoriness of Preferences in Matching and Choice. Foundations of Computing and Decision Sciences, 22(1), 3-19.

(11) Mussone, L. \& Sala, G. (2001). A Method to Evaluate Vehicular Accident Probability by Using Self Organizing Maps. CUPUM $-7^{\text {th }}$ International Computers in Urban Planning and Urban Management Conference, University of Hawaii at Monoa Honolulu, Hawaii, USA.

(12) Osgood, C.E.; Suci, G.J. \& Tannenbaum, P.H. (1957). The Measurement of Meaning. University of Illinois Press, Urbana, IL, USA.

(13) PROMON (1996). Modelo de Localização Integrada de Térmicas com Objetivos Múltiplos - Descrição Geral e Estudos de Caso. Manual do Usuário. 90p.

(14) Ramos, R.A.R. (2000). Localização Industrial - Um Modelo Espacial para o Noroeste de Portugal. Braga - Portugal. 299p. Tese (Doutorado), Universidade do Minho.

(15) Saaty, T.L. (1977). A Scaling Method for Priorities in Hierarchical Structures. Journal of Mathematical Psychology, 15(3), 234-281.

(16) Saaty, T.L. (1980). Analytical Hierarchy Process: Planning, Priority Setting, Resource Allocation. MCGraw-Hill, New York, NY, USA.

(17) Saaty, T.L. \& Vargas, L.G. (1991). Prediction, Projection and Forecasting. Kluwer Academic Publishers, Boston, MA, USA.

(18) Star, J. \& Estes, J. (1991). Geographic Information Systems: an Introduction. Englewoods Cliffs, New Jersey.

(19) Stillwell, W.G.; Seaver, D.A. \& Edwards, W. (1981). A Comparison of Weight Approximation Techniques in Multiattribute Utility Decision Making. Organization Behavior and Human Performance, 28(1), 62-77.

(20) von Winterfeldt, D. \& Edwards, W. (1986). Decision Analysis and Behavioural Research. Cambridge University Press, Cambridge, UK.

(21) Yager, R.R. (1988). On Ordered Weighted Averaging Aggregation Operators in Multicriteria Decision Making. IEEE Transactions on Systems, Man and Cybernetics, 8(1), 183-190.

(22) Zadeh, L.A. (1965). Fuzzy Sets. Information and Control, 8, 338-353.

(23) Zambon, K.L.; Carneiro, A.F.M.; Silva, A.N.R. \& Negri, J.C. (2003). Utilização de Mapas Auto-organizáveis para a Estimativa do Nível de Poluição em Processos de Alocação de Usinas Termoelétricas. VI SBAI - Simpósio Brasileiro de Automação Inteligente, Bauru, São Paulo, Brasil, 319-324. 